\title{
Moral hazard with random participation
}

\author{
Guillaume Roger * \\ The University of New South Wales
}

May 14, 2012

\begin{abstract}
This paper studies a principal-agent problem of moral hazard, in which the outside option is stochastic. This renders the agent's participation decision random from the perspective of the principal. The participation cost is no longer defined in terms of the agent's outside option but in terms of the principal's marginal benefit of participation. The optimal contract (i) entails information rents; (ii) features a trade-off between participation probability and rents and (iii) induces a lower effort than the standard model. Random participation results in weaker incentives and in twofold (ex ante) welfare losses. Menus of contracts (screening mechanisms) are not helpful to extract information because the single-crossing condition does not hold.
\end{abstract}

Keywords: moral hazard, asymmetric information, contract, participation constraint, principalagent. JEL Classification: D82,D86.

\section{Introduction}

The canonical model of moral hazard takes the agent's outside option as known to the principal designing the incentive contract. This simplifying assumption allows the analyst to focus attention on the incentive problem: the only friction stems from moral hazard, while participation can always be secured. This approach has spawn a vast literature and has been successfully extended to problems of moral hazard in teams (Holmström, 1982; Itoh,1993), multitasking (Holmström and Milgrom, 1991), hierarchies (Tirole, 1986) or even common agency (Bizer and DeMarzo, 1992;

*School of Economics, UNSW. Email: g.roger@unsw.edu.au. I owe special thanks to my colleagues Richard Holden, Chris Bidner and Suraj Prasad for most helpful comments. 
Martimort, 2004) and to countless applications. In doing so however the question of participation has been neglected; this is neither natural nor innocuous.

It is not natural because employers likely do not know their prospective hire's outside option, who may receive other offers from market participants. In financial contracting, it is unlikely that any one bank knows exactly other lenders' terms - i.e. the borrower's reservation utility. It is not innocuous because assuming that one knows the outside option is tantamount to disregarding information about the environment that is relevant to the optimal contract. In this paper I relax this standard assumption and let the outside option be stochastic. This random variable can be thought of as the reduced form of a game between principals competing for agents-say in a labor market. Allowing for the outside option to be uncertain sheds new light on the nature of the participation problem, which is found to interact subtly with the incentive problem.

Bar for the randomness of the outside option described by a common-knowledge distribution, the model is a standard principal-agent problem of moral hazard. The optimal contract still bears some of the standard properties; in particular, the transfer function is increasing in the output according to the likelihood ratio of the distribution (Holmström, 1979, Rogerson, 1985b, Jewitt 1988). The paper's central feature is a rent extraction-participation trade-off: conditional on participation, the principal wants to limit the transfer paid to the agent, but he also must offer high-enough a payment to secure said participation. Technically, the objective function becomes non-monotonic concave in the transfer $t$, while it is monotonically decreasing in the standard problem. ${ }^{1}$ The participation cost is thus not defined in terms of the agent's outside option, which is unknown. Rather it is exactly given by the principal's marginal benefit of securing participation, which always (necessarily) exceeds the agent's shadow cost (the Lagrange multiplier in the standard problem). That is, to secure participation the principal is willing to pay more (than in the standard problem) for any effort level. This interacts with the incentive problem in that the principal internalizes the higher cost of any given action; his preferred action is thus lower than the standard second-best. From a social standpoint this is costly on two accounts. First, the social surplus decreases when effort decreases. Second, with a lower action the agent's expected utility in equilibrium is also

\footnotetext{
${ }^{1}$ Put another way, any agent who accepts a contract necessarily receives an ex ante rent (except for the marginal agent). The principal would like to extract this rent but can do so only imperfectly because the agent chooses her action, not the principal.
} 
lower. Therefore the probability of participation decreases and gains from trade are lost.

With the characterization in hand two sets of comparative statics can be derived. The first one concerns itself with the impact of a change in the the incentive problem (i.e. distribution of output and agent's risk aversion) on the participation probability. More dispersion of the distribution output, or more risk aversion, both lead to a lower participation threshold. In the second set of comparative statics I study the effects of changes in the contracting environment captured by changes in the distribution of the outside option. A shift the distribution in the sense of firstorder stochastic dominance unambiguously results in a lower action being induced, and therefore in a lower participation probability. A mean-preserving spread of the same distribution has more ambiguous consequences.

A natural question that arises is whether the principal may be made better off by using menus of incentive contracts. Indeed a revelation mechanism may be called on to elicit the agent's private information about her outside option. If so, the principal would essentially trade-off an information rent (under the revelation mechanism) for another (without it). Truthful revelation of that information can only be achieved with non-contingent transfer functions; that is, menus are useless. The reason is that, unlike in a standard adverse selection problem, there is no direct (i.e. technology or preferences) connection between the agent's private information (here the outside option) and her choice of action. In technical terms, the single-crossing condition does not hold in a strong sense: the game is not supermodular in the agent's private information.

As a by-product of the analysis, the linear contract derived by Holmström and Milgrom (1987) is shown to be a very special case. In that model the contract divorces participation from incentives. With a stochastic outside option, this results in the slope of the linear contract, and therefore the equilibrium action, to remain unchanged. This insight is in fact quite misleading, and unique to the exponential specification.

This paper bears a nominal connection to a paper by Rochet and Stole (2000), who study random outside option in the context of adverse selection. The analysis here turns out to be simpler and has a different economic content. Broadly speaking I find results that run counter to theirs. Under adverse selection, uncertainty of the outside option lowers the probability of paying the information rent, so distortions used to decrease that rent can be relaxed. Under moral hazard, uncertainty of the reservation utility increases the cost of any one action, so it creates incentives 
for further distortions.

The literature on moral hazard is vast and rich. This work builds on the well-known contributions of Holmström (1979), Rogerson (1985b) and Jewitt (1988), who study what has become the standard moral hazard problem. Beyond that however, perhaps surprisingly, no other paper combines moral hazard and stochastic outside option. Somewhat tangentially, this paper relates to the work of Laffont and Tirole (1986), who analyze a combination of adverse selection and moral hazard. Their revelation mechanism has bite because the agent's private information directly affects her payoffs, unlike here. Conditional on extracting that private information, the optimal action is not distorted (conditionally first-best). In the present model, the optimal effort is "conditionally second-best": the distortion is indirect, as in Laffont and Tirole (1986), and in response only to the modified transfer. This study also connects to the works of Lewis and Sappington (1989), Maggi and Rodriguez-Clare (1995) and Jullien (2000) who investigate the role of type-dependent reservation utility in models of adverse selection. When the outside option is high enough, the interaction of the participation constraint and the incentive constraint generates countervailing incentives that may reverse the direction of the usual distortions. The nature of the present work is quite different but it is worth noting that it is precisely the interaction of the moral hazard constraint and the participation probability, through the transfer, that is the source of distortions.

The next Section introduces the model. In Section 3, I start with the standard model to fix ideas and conduct most of the analysis. Section 4 discusses the linear model Holmström and Milgrom (1987) and Rochet and Stole's (2000) work. Last I conclude. All proofs are sent to the Appendix.

\section{Model}

A principal delegates a task to an agent. She undertakes an unobservable action $a \in \mathcal{A} \subset \mathbb{R}_{+}$. The action yields a stochastic outcome $q \in[\underline{q}, \bar{q}] \equiv \mathcal{Q} \subset \mathbb{R}_{+}$with conditional distribution $F(q \mid a)$ and corresponding density $f(q \mid a)>0$, at cost $c(a)$ increasing and convex. The density $f(q \mid a)$ satisfies the MLRP: $f_{a}(q \mid a) / f(q \mid a)$ is increasing, concave in $q$; therefore $F\left(q \mid a^{\prime}\right)$ stochastically dominates $F(q \mid a)$ in a first-order sense when $a^{\prime}>a$. Upon a realization of $q$, the agent receives a transfer $t$. Her net utility is given by $v(t, a) \equiv u(t)-c(a)$, where $u: \mathbb{R} \mapsto \mathbb{R}$ is a continuous, increasing, concave function. There is no limited liability constraint. Throughout the principal can commit 
to the contract and receives a net payoff $S(t ; q)=q-t$. Said contract $\mathcal{C}=\langle t(q), a\rangle$ is an effort recommendation $a$ and a transfer function $t: \mathcal{Q} \mapsto \mathbb{R}$.

For that contract to be accepted by the agent, it must satisfy an individual rationality constraint. The object of this paper is to study the effects of randomness at that stage of the game. The participation decision itself is not random, of course; rather the outside option is. Specifically, the agent's outside option $u_{0}$ follows a log-concave, common knowledge distribution $G\left(u_{0}\right), u_{0} \in \mathcal{U}_{0} \equiv$ $\left[\underline{u}_{0}, \bar{u}_{0}\right] \subset \mathbb{R}$. That $\mathcal{U}_{0}$ be bounded is without consequences on the results. That outside option is unknown to the principal; it is private to the agent when she decides whether to accept the contract. The timing is otherwise standard:

1. The principal offers a contract $\mathcal{C}$;

2. The outside option $u_{0}$ is realized. It is known to the agent only; ${ }^{2}$

3. The agent accepts or rejects the contract. If rejecting, she receives her outside option and the principal gets 0 . If accepting, she also chooses an action $a$;

4. Action $a$ generates an outcome $q \in \mathcal{Q}$;

5. Transfers are implemented and payoffs are realized.

\section{Analysis}

\subsection{The standard problem}

In the standard model, the principal's programme reads

\section{Problem 1}

$$
\max _{t, a} \int_{\mathcal{Q}}[x-t(x)] d F(x \mid a)
$$

s.t.

$$
a \in \arg \max _{a^{\prime} \in \mathcal{A}} \int_{\mathcal{Q}} u(t(x)) d F\left(x \mid a^{\prime}\right)-c\left(a^{\prime}\right)
$$

and

$$
U(t, a) \equiv \int_{\mathcal{Q}} u(t(x)) d F(x \mid a)-c(a) \geq u_{0}
$$

\footnotetext{
${ }^{2}$ Whether stages 1 and 2 are interchanged has no bearing on the outcomes of the game.
} 
for some exogenous, known outside option $u_{0}$. Let $\left(a^{S B}, t^{S B}\right)$ denote the solution to Problem 1 . This is a well known problem, studied under different angles and with different motivations, by Mirrlees (1975, 1999), Holmström (1979), Rogerson (1985b), Jewitt (1988), Araujo and Moreira (2001) or Conlon (2009). Typically the participation (3.2) constraint binds - except if the agent is subject to a limited liability constraint. Jewitt (1988) and Conlon (2009) have also provided tractable conditions for the first-order approach (FOA) to be valid; these conditions are assumed to hold in this paper.

\subsection{Random participation}

Let $\tilde{u}_{0}$ be a realization of $u_{0}$. When $u_{0} \sim G\left(u_{0}\right)$, the participation constraint of the agent is satisfied with probability $\operatorname{Pr}\left(U(t, a) \geq \tilde{u}_{0}\right) \equiv G((U(t, a))$. This immediately gives us a first result.

Lemma 1 In any contract accepted by the agent, she receives an ex ante rent; i.e. whenever (3.2) is satisfied, $U(t, a)>\tilde{u}_{0}$ for any realization of $u_{0}$, except on a set of measure 0 .

The proof is immediate and therefore omitted. Lemma 1 informs us that the agent's participation cannot bind. With this Problem 1 rewrites as:-

\section{Problem 2}

$$
\max _{t, a} G((U(t, a))) \int_{\mathcal{Q}}[x-t(x)] d F(x \mid a)
$$

s.t.

$$
\frac{\partial U(t, a)}{\partial a} \equiv \int_{\mathcal{Q}} u(t(x)) d F_{a}(x \mid a)-c^{\prime}(a)=0 ; \forall U(t, a) \geq \tilde{u}_{0}
$$

Let $\pi(t, a) \equiv \int_{\mathcal{Q}}[x-t(x)] d F(x \mid a)$ and $U=U(t, a)$ for ease of notation. Attach multiplier $\mu$ to (3.3). A solution to this problem must satisfy the first-order conditions:-

$$
\frac{1}{u^{\prime}(t)}=\frac{g(U)}{G(U)} \pi(t, a)+\mu \frac{f_{a}(q \mid a)}{f(q \mid a)}
$$

and

$$
\int_{\mathcal{Q}}[x-t(x)] d F_{a}(x \mid a)+\mu\left[\int_{\mathcal{Q}} u(t(x)) d F_{a a}(x \mid a)-c^{\prime \prime}(a)\right]=0
$$

by application of the Envelop Theorem (i.e. (3.3) holds). Let $t^{*}$ solve (3.4) and $a^{*}$ be a solution to (3.5); let them induce $U\left(t^{*}, a^{*}\right)=U^{*}$ and denote by $u_{0}^{*}$ the value of $u_{0}$ for the marginal participating agent. I make three claims about these conditions. First, 
Lemma 2 At a solution of Problem 2 the principal is effort constrained, i.e. the multiplier $\mu$ is strictly positive.

An immediate consequence of Lemma 2 is that the transfer function $t(q)$ is increasing, concave in $q$ as in the standard contract thanks to the MLRP. Second,

Lemma 3 Conditions (3.4) and (3.5) are (necessary and) sufficient to identify a solution of Problem 2.

and third,

Lemma $4 U^{*}>\underline{u}_{0}$.

This last Lemma essentially asserts that the optimal contract does not induce a trivial solution. That is, the transfer $t^{*}(q)$ is bounded according to (3.4), some effort is exerted in that $a^{*}>\min a$ by (3.5) and a positive measure $G\left(U^{*}\right)$ of agents may participate.

Condition (3.4) departs in an obvious way from the standard first-order conditions characterizing $t^{S B}$ in Problem 1. The term $\frac{g(U)}{G(U)} \pi(t, a)$ appears in lieu of the standard Lagrange multiplier. Condition (3.5) is thus also altered, however indirectly through the transfer $t$, which enters the first-order condition (3.5) and therefore determines the optimal action $a^{*}$. In the spirit of Laffont and Tirole (1986), this contract can be called "conditionally second-best": the action is not directly distorted, but the transfer is - with consequences on the equilibrium action. ${ }^{3}$

We will return to the interpretation of these conditions. For now I note that the hazard rate $g / G$ appears. With this in mind,

Proposition 1 The threshold $U^{*}$ is unique. For all $u_{0} \leq U^{*}$ the agent participates, while she does not if $u_{0}>U^{*}$.

In the first-order condition (3.4), the hazard rate $g\left(U^{*}\right) / G\left(U^{*}\right)$ exactly identifies the rent $U^{*}$ that defines the participation threshold. This truncated density multiplies the principal's expected profit. That is, a marginal increase in the agent's rent increases the probability of acceptance, in which case the principal receives $\pi(t, a)$. Indeed $\frac{g\left(U^{*}\right)}{G\left(U^{*}\right)} \pi(t, a)$ is the marginal benefit of increasing $t$ to secure the agent's participation. Importantly, Condition (3.4) tells us that the participation

\footnotetext{
${ }^{3}$ In that paper, the effort is "conditionally first-best" but the allocation is distorted to solve an adverse selection problem.
} 

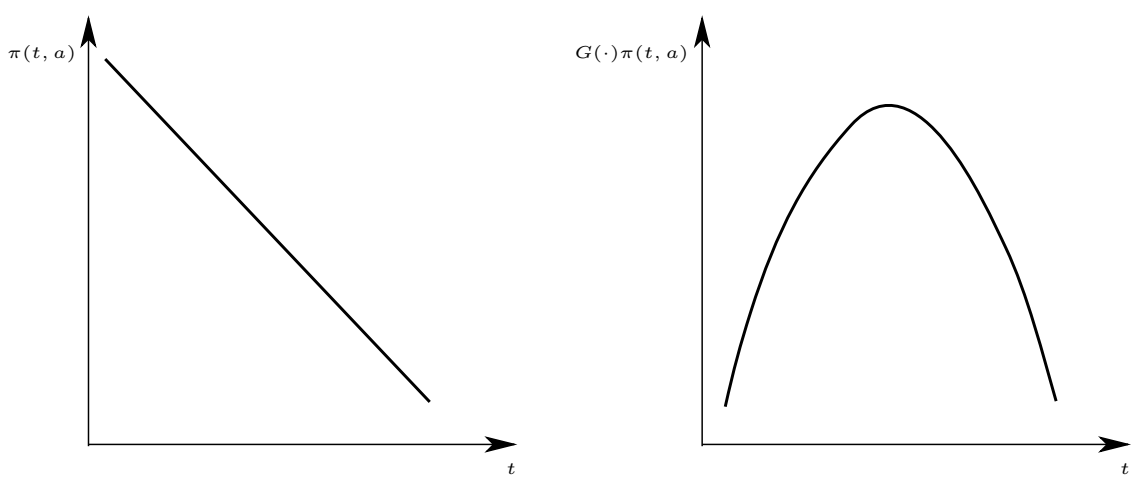

Figure 1: objective functions. Problem 1 (left) and Problem 2 (right)

cost is not defined in terms of the outside option $u_{0}$, as it is in Problem 1; that outside option is unknown. ${ }^{4}$ Rather the cost of participation is determined in terms of the principal's marginal benefit of securing participation. In this model the participation problem induces a rent extractionparticipation trade-off between securing participation and offering excessive rents. This comes about because not assuming that $u_{0}$ is known drastically modifies the principal's objective function. In Problem 1 it is monotonically decreasing in $t$ (for a fixed action $a$ ); in contrast, in Problem 2 it is non-monotonic and concave in $t$ - hence the trade-off. ${ }^{5}$

\subsection{Properties of the optimal contract}

Lemmata 1, 3 and 4 and the first-order condition (3.4) already inform us that the optimal contract entails some distortion away from the standard second best. Because there are (ex ante) rents (by Lemma 1), the transfer is necessarily "too high" as compared to that of the standard problem (where the participation constraint binds). The solution $a^{*}$ of Condition (3.5) is therefore also distorted, even if only indirectly, compared to $a^{S B}$. I want to formalize these ideas.

First it can be easily shown that the principal overpays the agent for any given action, as compared to the standard problem (Problem 1).

Lemma 5 Fix the action $a$. For any realization $u_{0} \leq u_{0}^{*}, \forall q, t^{*}(q)>t^{S B}(q)$.

In light of Lemma 1 this result may not be surprising; that is, overpaying the agent contributes to

\footnotetext{
${ }^{4}$ Recall that in Problem 1 Constraint (3.2) has a multiplier $\lambda\left(u_{0}\right)>0$, which is its shadow cost.

${ }^{5}$ Pointwise differentiation of the objective function yields $[g(U) / G(U)] \cdot u^{\prime} \cdot \pi(t, a)-1$. Differentiating again yields a negative expression by $\log$-concavity of $G$ and concavity of $u(t)$.
} 
generating the rent $U^{*}$ identified in Lemma 1. But it is not the only channel through which rents may be created: the action may also be chosen so as to leave the agent with a rent. Thus Lemma 5 does identify the source of the rent as being a more generous transfer for any given action. The fundamental reason is that the principal's problem is quite different when the outside option is stochastic, as previously discussed. Lemma 5 also implies that participation is more costly to the principal with a random outside option than in Problem 1. To see why, fix $a$, multiply both sides of the FOC (3.4) by $f(q \mid a)$ and integrate over $\mathcal{Q}$ to find:-

$$
\mathbb{E}_{q}\left[\frac{1}{u^{\prime}\left(t^{*}\right)}\right]=\frac{g}{G} \pi(t, a)
$$

Similarly with the standard FOC of Problem 1:-

$$
\mathbb{E}_{q}\left[\frac{1}{u^{\prime}\left(t^{S B}\right)}\right]=\lambda
$$

so $t^{*}(q)>t^{S B}(q) \forall q \Rightarrow \frac{g}{G} \pi(t, a)>\lambda$, i.e. the cost of participation is larger for a given action.

Given that he overpays (for any fixed action $a$ ), the principal has incentives to implement some distortion to further limit the rent $U^{*}$ left to the agent, but is unable to. The reason is that, no matter how one may go about it, further rent extraction necessarily implies effort distortion, but that decision is not the principal's to make. To see where these incentives stem from, by Lemma 4 the expected cost of the contract is

$$
T \equiv \int_{\underline{u}_{0}}^{u_{0}^{*}} \int_{\mathcal{Q}} t(x ; z) d F(x \mid a) d G(z)
$$

Now let

$$
t\left(a ; u_{0}\right) \equiv \int_{\mathcal{Q}} t\left(x ; u_{0}\right) d F(x \mid a)
$$

Because $t\left(q ; u_{0}\right)$ is increasing, concave in $q$, we know from Conlon $(2008$, Lemma 1$)$ that $t\left(a ; u_{0}\right)$ is non-decreasing, concave in $a$. Therefore (3.6) rewrites

$$
T(a) \equiv \int_{\underline{u}_{0}}^{u_{0}^{*}} t(a ; z) d G(z)
$$

which is an increasing function of $a$. So reducing the principal's expected cost requires the effort to drop. However the principal is in no position to impose an effort distortion: unlike in a problem of adverse selection, he does not choose the allocation. Rather, given a contract, the agent does through her action $a$. This is subgame-perfection at work, which is captured by the application of the Envelop Theorem when deriving (3.5) - i.e. given any $t, \partial U(t, a) / \partial a=0$. 


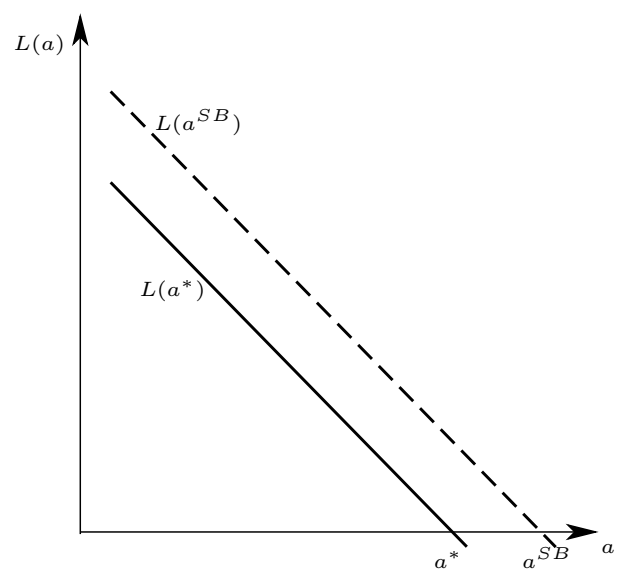

Figure 2: the principal's first-order condition (3.5).

Lemma 5 takes the action $a$ as fixed; of course it is endogenous too. The next Proposition presents a cross-model comparison of the equilibrium.

Proposition 2 Fix the economy $\mathcal{Q}, F(q \mid \cdot), c(\cdot), \mathcal{U}_{0}, G\left(u_{0}\right)$. For any contract that the agent accepts under random participation,

1. the effort is lower than in the standard problem; and

2. the transfer function $t^{*}(q)$ lies everywhere (in $\left.\mathcal{Q}\right)$ below the second-best $t^{S B}(q)$;

i.e. $\forall \tilde{u}_{0} \leq u_{0}^{*}, a^{*}<a^{S B}$ and $t^{*}(q)<t^{S B}(q) \forall q \in \mathcal{Q}$.

Facing a higher cost for any given action (Lemma 5) the principal necessarily prefers a lower action than the second-best. This is shown in Figure 2. This in turns moderates the expected cost $T(a)$. To see why, consider an exogenous increase in the transfer; differentiate (3.5) with respect to $t$ :

$$
-\int_{\mathcal{Q}} d F_{a}+\mu \int_{\mathcal{Q}} u^{\prime} d F_{a a}+S O C \frac{d a}{d t}+\frac{d \mu}{d t}\left\{\int_{\mathcal{Q}}[x-t(x)] d F_{a}+\mu\left[\int_{\mathcal{Q}} u(t(x)) d F_{a a}-c^{\prime \prime}\right]\right\}=0
$$

where SOC is the second-order condition of (3.5). The last term is zero, therefore $\frac{d a}{d t}<0$ from the perspective of the principal. In turn this adjustment has implications the agent: offering a transfer that induces her to decrease her action below $a^{S B}$ not only decreases the agent's expected utility by first-order stochastic dominance, it also increases the risk. ${ }^{6}$ Proposition 2 paves the way for a reasonably intuitive Corollary.

\footnotetext{
${ }^{6}$ MLRP implies first-order stochastic dominance, as noted earlier, which is sufficient for second-order stochastic dominance. Hence the distribution $F\left(q \mid a^{*}\right)$ is more risky than $F\left(q \mid a^{S B}\right)$.
} 
Corollary 1 Take some $\bar{u}_{0} \in \mathcal{U}_{0}$ such that $U^{S B}=\bar{u}_{0} ; u_{0}^{*}<\bar{u}_{0}$

In other words, a further consequence of a stochastic outside option is that the participation probability is lower than it would be under the second-best contract. The reason for a decrease in participation between these two contracts is quite simple: a lower action always decreases the rent $U^{*}(a) \equiv \int_{Q} u\left(t^{*}(x)\right) d F\left(x \mid a^{*}\right)-c\left(a^{*}\right)$ of the agent (even across models here). Therefore the participation condition (3.2) becomes more difficult to satisfy.

Together Proposition 2 and Corollary 1 speak to the extent of the interaction between the random nature of the outside option and the endogenous variables of the contract. The important implication of Proposition 2 and Corollary 1 is that moving from a deterministic to a stochastic reservation utility has twofold consequences on welfare. First the optimal action is lower; second the probability that a welfare-enhancing relationship is entered into, decreases.

\subsection{Comparative statics}

This model affords the opportunity to perform an interesting comparative statics exercise on two exogenous elements: the distribution $F(q \mid a)$ (as well as the agent's risk aversion), and the distribution $G\left(u_{0}\right)$, which summarizes the contracting environment. Namely I want to understand how changes in $F(q \mid a)$ (or the utility function $u(\cdot)$ ) affect the participation threshold $u_{0}^{*}$, which is governed by $G\left(u_{0}\right)$. Conversely, I am interested in understanding how changes in the environment, described by properties of the distribution of $u_{0}$, interact with the moral hazard problem. These are the object of the next two Propositions.

Proposition 3 The transfer $t^{*}$, the optimal action $a^{*}$ and participation (i.e. $G\left(U^{*}\right)$ ) all decrease in the dispersion of the distribution $F(q \mid a)$ and in the agent's risk-aversion.

That $t^{*}$ and $a^{*}$ decrease with dispersion and risk aversion is not puzzling. The third claim is less obvious. Note that $U(a)$ is decreasing in both dispersion of $F(q \mid a)$ and risk aversion. ${ }^{7}$ Therefore the hazard rate $g(U) / G(U)$ increases, which contributes to increasing $t^{*}$ by (3.4). However this need to secure participation (by raising $t^{*}$ ) is dominated by the incentive problem: any $q$ becomes a less informative signal of the agent's effort when $F(q \mid a)$ is more dispersed.

\footnotetext{
${ }^{7}$ This differs from Corollary 1 and its commentary. There I compare participation probabilities across models; here I consider the contract under random outside option only and let the distribution $F(q \mid a)$ vary exogenously.
} 
Proposition 3 considers the impact of the agent's incentive problem on participation. The next set of results operate differently: starting from changes in the outside option, which is exogenous to the agent's behavior (i.e. to solving (3.3)), I ask what it implies for the principal's choices. Of course the principal's contract offer, which depends on $G\left(u_{0}\right)$, ultimately affects the agent's action.

Proposition 4 Take two distributions $G^{1}\left(u_{0}\right)$ and $G^{2}\left(u_{0}\right)$.

1. Suppose that $G^{1}$ first-order stochastically dominates $G^{2}$; the action $a^{*}$ solving (3.5) and participation (i.e. $G\left(U\left(a^{*}, u_{0}\right)\right)$ ) are both lower under $G^{1}$ than $G^{2}$.

2. Suppose that $G^{2}$ is a single mean-preserving spread of $G^{1}$, and let $u_{0}^{\prime}$ denote the realization such that $G^{2}\left(u_{0}^{\prime}\right)=G^{1}\left(u_{0}^{\prime}\right)$. When $u_{0}^{*}<u_{0}^{\prime}$ the action $a^{*}$ solving (3.5) and participation are both lower under $G^{1}$ than $G^{2}$. Conversely when $u_{0}^{*}>u_{0}^{\prime}$.

If recalling Proposition 2 and Corollary 1, the second claim follows intuitively: take a perfectly known outside option and introduce a little uncertainty around it, Proposition 2 suggests that effort decreases and Corollary 1 that participation drops. Proposition 4 formalizes this insight.

Both these claims rely on the behavior of the hazard rate $g / G$. To gain some intuition, recall the first-order condition (3.4) where, ceteris paribus, the transfer increases in the hazard rate; this corresponds to a smaller $G$. Therefore the cost of a given action is higher under $G^{1}$ than $G^{2}$. The difference between the two claims of Proposition 4 is this: under first-order stochastic dominance $G^{1} \leq G^{2}$ everywhere, so the ordering of the hazard rates never changes. This is not true under a mean-preserving spread. At $u_{0}^{\prime}$ the ordering of the distributions changes and so does that of the hazard rates. The transfer decreases because its marginal impact on the participation decision is low: when $u_{0}^{*}>u_{0}^{\prime}$, participation is already secured for most realisations of the outside option. ${ }^{8}$

\subsection{Menus of contracts}

Faced with some hidden information one may wonder whether the principal could not do better by using menus to screen the agent's outside option $u_{0}$ (her type). It turns out that menus cannot make the principal better off in this model. Suppose he uses a direct revelation mechanism to elicit the agent's private information of the form $\left\langle t\left(q ; u_{0}\right), a\left(u_{0}\right)\right\rangle$. Given a message $u_{0}$, that contract

\footnotetext{
${ }^{8}$ The restriction to single mean-preserving spread is for convenience only; then $G^{1}$ and $G^{2}$ cross only once.
} 
is the standard second-best contract corresponding to Problem 1. We know from the standard first-order condition $1 / u^{\prime}=\lambda\left(u_{0}\right)+\mu^{S B} f_{a} / f$ that $t\left(q ; \tilde{u}_{0}\right) \neq t\left(q ; \hat{u}_{0}\right)$ for $\tilde{u}_{0} \neq \hat{u}_{0}$. From (3.1) it then follows that $a\left(\tilde{u}_{0}\right) \neq a\left(\hat{u}_{0}\right)$. Now consider a truth-telling constraint

$$
U\left(t\left(\cdot ; \tilde{u}_{0}\right), a\left(\tilde{u}_{0}\right), \tilde{u}_{0} ; \tilde{u}_{0}\right) \geq U\left(t\left(\cdot ; \hat{u}_{0}\right), a\left(\hat{u}_{0}\right), \hat{u}_{0} ; \tilde{u}_{0}\right), \forall \tilde{u}_{0}, \hat{u}_{0}
$$

when the agent is of type $\tilde{u}_{0}$ but may report some $\hat{u}_{0}$. This condition is equivalent to saying that the agent solves

$$
\hat{u}_{0} \in \arg \max _{u_{0} \in \mathcal{U}_{0}} U\left(t\left(\cdot ; \hat{u}_{0}\right), a\left(\hat{u}_{0}\right), \hat{u}_{0} ; \tilde{u}_{0}\right) ;
$$

or, differentiating at $\hat{u}_{0}=\tilde{u}_{0}$, means

$$
\left.\frac{\partial U}{\partial t} \frac{\partial t}{\partial u_{0}}\right|_{\tilde{u}_{0}}+\left.\frac{\partial U}{\partial a} \frac{\partial a}{\partial u_{0}}\right|_{\tilde{u}_{0}}=0
$$

By (3.3), $\frac{\partial U}{\partial a}=0$ and by monotonicity of $u(\cdot), \frac{\partial U}{\partial t}>0$. Therefore truthful revelation requires $\left.\frac{\partial t}{\partial u_{0}}\right|_{\tilde{u}_{0}}=0$ : there can be no discrimination on the basis of the outside option. But then eliciting that information is useless. Thus we have

Proposition 5 A menu of contracts contingent on the agent's outside option cannot do better than the (single) non-linear contract given by (3.4) and (3.5).

Why this result? Unlike in a standard screening model, here the agent takes an action $\hat{a}$ given $t\left(q ; u_{0}\right)$ after sending a message $\hat{u}_{0}$. That action is necessarily optimal; this is subgame perfection at work, and the second term of (3.9). Moreover, the single-crossing property does not hold - this is the first term of (3.9). ${ }^{9}$ The reason is that there is no direct connection between the agent's type (her outside option $u_{0}$ ) and her marginal utility of $q \cdot{ }^{10}$ Hence all types pool. Proposition 5 leads to an immediate Corollary.

Corollary 2 A participation fee cannot be used to extract the agent's rent.

for which the proof is evident and therefore omitted. Relying on the agent's message to set a participation fee is impossible by Proposition 5. At the same time, charging a(n) (expected) participation fee based on the measure of participating agents $\int_{\underline{u}_{0}}^{u_{0}^{*}} d G(z)$ would precisely deter the marginal agent(s).

\footnotetext{
${ }^{9}$ The single-crossing condition is lost in a broad sense: the game is not even supermodular.

${ }^{10}$ Of course, Propositions 2-4 make clear some connection does exist, but it is indirect; i.e. through the transfer $t^{*}$ and the FOC (3.5).
} 
Remark 1 An alternative timing may be to let the agent decide whether to accept the contract before she knows her outside option. In an adverse selection context, this is known as ex ante contracting. It is easy to see that the standard solution prevails, where $u_{0}$ is replaced by $\mathbb{E}\left[u_{0}\right]$ and $\lambda \equiv \lambda\left(\mathbb{E}\left[u_{0}\right]\right), \mu \equiv \mu\left(\mathbb{E}\left[u_{0}\right]\right)$.

\section{Discussion}

\subsection{The linear model}

The CARA-normal-linear framework of Holmström and Milgrom (1987) has proven to be a very useful model for much applied work. In a dynamic environment, the linear contract has also been shown to be optimal - under some restrictions (Holmström and Milgrom (1987)). In this section it is shown that the linear model offers insights about the impact of randomness of the outside option that do not extend to a more general setting.

Let $t=\alpha+\tau q$ be the tariff offered, $c(a)=(c / 2) a^{2}$ and $u=-e^{r(t-c(a))}$, where $r$ is the coefficient of risk aversion. Let also $q \sim \mathcal{N}\left(0, \sigma^{2}\right)$. We know that the agent's problem upon accepting the contract (i.e. (3.3)) is unchanged, so that the principal's program can be directly written

\section{Problem 3}

$$
\max _{\alpha, \tau} G\left(-e^{-r\left[\alpha+\tau^{2} / 2 c-(r / 2) \tau^{2} \sigma^{2}\right]}\right)\left[\frac{\tau}{c}-\left(\alpha+\frac{\tau^{2}}{c}\right)\right]
$$

Some optimization and simple algebra leads to the standard solution for the slope: $\tau=1 /\left(1+r c \sigma^{2}\right)$. Because the slope of the affine contract in unchanged, the agent's optimal action is unaffected and remains as in the second-best problem. In this model therefore the stochastic nature of the outside option has no consequences on incentives, and therefore on welfare.

This outcome is a feature of the exponential specification. The term $\tau$ is independent of $u_{0}$, which is not true according to the standard condition $1 / u^{\prime}=\lambda+\mu^{S B} f_{a} / f$ of Problem 1 (since $\lambda \equiv \lambda\left(u_{0}\right)$ in particular, and even less when $g / G$ enters the FOC (3.4) directly). The reason is that in the Holmström-Milgrom model (1987), the agent's optimal action defined as $a=\tau / c$ is independent of level of utility, unlike in (3.1) or (3.3). Consequently, the rate of substitution of the principal between instruments $\alpha$ and $\tau$ is also independent of level of utility. Indeed, take the 
first-order conditions of the standard linear-CARA-normal problem

$$
\begin{aligned}
-1+\lambda r e^{-r[\cdot]} & =0 \\
\left(\frac{1}{c}-2 \frac{\tau}{c}\right)+\lambda r e^{-r[\cdot]}\left(\frac{\tau}{c}-\tau r \sigma^{2}\right) & =0
\end{aligned}
$$

Re-arranging and dividing one by the other gives the "marginal rate of substitution" between $\alpha$ and $\tau$, which is independent of $e^{-r[\cdot]}$. In consumer theory this leads to corner solutions. The "corner solution" here is to always increase $\alpha$ to satisfy participation, while $\tau$ remains unchanged. In other words, the exponential utility does not just "abstract from wealth effects" (as noted by Holmström and Milgrom); there is complete, but somewhat artificial, separability of the instruments available to the principal. A similar property can be verified when the outside option is random; $\tau$ never changes while $\alpha$ adjusts to solve the participation problem as in Problem 2. Then the comparative statics of Proposition 3 all carry over, where dispersion is measured by the variance but Proposition 4 no longer follows in it entirety as effort does not change.

To further the insight into the limitations of the CARA-linear-normal model regardless of whether the outside option is random, consider the following simple example. Take a linear contract $t=\alpha+\tau q$, an effort cost $c(a)=a^{2} / 2$, a simple two-outcome space: $q \in\{0,1\}$ with a distribution $\operatorname{Pr}(q=1)=a$ and an arbitrary utility function $u(\cdot)$, increasing and concave. The agent's payoff is

$$
a u(\alpha+\tau)+(1-a) u(\alpha)-\frac{a^{2}}{2}
$$

so that the maximizer is $\hat{a}=u(\alpha+\tau)-u(\alpha)$. Internalizing this, the principal's payoff reads

$$
\hat{a}(1-\alpha-\tau)-(1-\hat{a}) \alpha
$$

which is maximized when $\tau^{*} \equiv \tau^{*}(\alpha)=1-\frac{u(\alpha+\tau)-u(\alpha)}{u^{\prime}(\alpha+\tau)}$. The optimal slope is a function of the intercept. The implication is this: with any other functional form than exponential utility, the intercept does affect the determination of the slope parameter, which governs incentives. So, the participation constraint is not neutral on incentives. With a stochastic outside option, participation is more costly to ensure, with now well understood consequences on the provision of incentives.

\subsection{Risk aversion of the principal}

One may conjecture that a risk-averse principal may want to increase the transfer function $t^{*}$ even further (for any $q$ ) in order to secure the agent's participation. When the principal is risk-averse 
the contract differs from the scheme characterized by (3.4) and (3.5) only to the extent of risk aversion. Indeed, let the principal have payoffs $w(q-t)$ with $w(\cdot)$ increasing and concave, the first order conditions now read:-

$$
\frac{w^{\prime}(q-t)}{u^{\prime}(t)}=\frac{g(U)}{G(U)} \pi(t, a)+\mu \frac{f_{a}(q \mid a)}{f(q \mid a)}
$$

and

$$
\int_{\mathcal{Q}} w(x-t(x)) d F_{a}(x \mid a)+\mu \int_{\mathcal{Q}} u(t(x)) d F_{a a}(x \mid a)-c^{\prime \prime}(a)=0
$$

The interpretation of (4.1) does not depart much from that of (3.4), but is quite illustrative: a very risk-averse principal wants at least some output; he will offer a flat transfer that secures participation and needs not induce high effort.

\subsection{Connection to Adverse Selection}

Bar for the appearance of the hazard rate $g(U) / G(U)$ in the first-order condition (3.4), the results of this paper bear little resemblance to those of Rochet and Stole (2000). This should come as little surprise in that adverse selection and moral hazard are quite different problems. It is worth mentioning however that Rochet and Stole (2000) show that the introduction of a random outside option reduces distortions (i.e. is welfare improving, most notably at the lower bound of the type space). In contrast I find that a random outside option enhances distortions (i.e. decreases welfare).

The ultimate reason for this essential difference resides in the fundamentally different nature of the agent's informational rents. In the adverse selection model the information rent exists regardless of the stochastic nature of the outside option. The principal pays that rent with probability $G(U)<1$ : randomness decreases the expected rent paid out. Reducing the distortion, which is costly to the principal, is thus inexpensive, as well as effective, to increase the probability of acceptance. Under moral hazard instead, the randomness of the outside option introduces a new informational rent that the principal can control only imperfectly using the transfer; no direct distortion of the allocation-here the action-is possible (recall (3.5)). More obviously, randomness of the outside option increases the expected $\operatorname{cost} T(a)$ of any action. Hence the principal's preferred action can only be lower than the second-best. 


\section{Conclusion}

This paper presents a model of moral hazard when the agent's reservation utility is uncertain. The introduction of a random outside option has real implications for the optimal contract. Securing participation becomes an issue for the principal, in addition to addressing the moral hazard problem; this introduces a new trade-off between rent extraction and participation. The optimal transfer reflects these twin concerns, and is costlier. This has consequences on his ability to provide incentives for effort: given a higher cost, the prescribed action is necessarily lower. Thus private information held by the agent about her outside option leads to a socially worse outcome. The welfare losses are in fact twofold: the principal prescribes a lower action, the consequence of which is a lower participation probability. This stands in contrast to the case of adverse selection, where that same private information is welfare enhancing in that distortions are reduced.

Any participating agent receives an ex ante rent in this model. One may thus interpret randomness of the outside option as akin to conferring some bargaining power to the agent. This would not be faithful to the details of the game; here the principal still makes a take-it-or-leave-it offer to the agent. In doing so however he is less informed than in the standard problem and thus faces the trade-off we now know.

Describing the outside option as a random variable can be conceived of as a reduced form for a market game, in which principals compete by posting contract and agents make participation and effort decisions. The current results lead me to conjecture that agents will receive rents and that contracts will display weaker incentives (than the second-best). While economists think of competition as being socially beneficial because it removes distortions, the present paper suggests the details of the competitive process and the contracting game are essential in achieving allocative efficiency. Furthermore, if the outside option $u_{0}$ is generated by offers from competing principals of varying quality (modeled for example as different supports $\mathcal{Q}$, or different transformations of $q$ ), the result herein seem to imply that lower-quality principals would only be able to induce lower effort than high types. That is, the moral hazard problem may amplify the differences in the principals' underlying abilities. The details of these questions are left for future research. 


\section{Appendix}

Proof of Lemma 2: Taking the FOC of the Lagrangean formed by the objective function and the constraint (3.3) is straightforward and therefore omitted. Both (3.4) and (3.5) arise by application of the Envelop Theorem (i.e. (3.3) holds). To show that $\mu>0$ I apply the proof of Jewitt (1988) to Conditions (3.4) and (3.3). Rewrite $f_{a}=\left(1 / u^{\prime}-[g(U) / G(U)] \pi(t, a)\right) f(q \mid a) / \mu$ from (3.4) and substitute in (3.3):

$$
\int_{\mathcal{Q}} u(t(x))\left(\frac{1}{u^{\prime}}-\frac{g(U)}{G(U)} \pi(t, a)\right) d F(x \mid a)=\mu c^{\prime}(a)
$$

Integrate (3.4) over $\mathcal{Q}: \mathbb{E}_{q}\left[1 / u^{\prime}\right]=\int_{\mathcal{Q}}[g(U) / G(U)] \pi(t, a) d F=[g(U) / G(U)] \int_{\mathcal{Q}} \pi(t, a) d F=$ $[g(U) / G(U)] \pi(t, a)$ by the Law of Iterated Expectations. Therefore

$$
\int_{\mathcal{Q}} u(t(x))\left(\frac{1}{u^{\prime}}-\mathbb{E}_{q}\left[\frac{1}{u^{\prime}}\right]\right) d F(x \mid a)=\mu c^{\prime}(a)>0
$$

so $\mu>0$ as claimed.

Proof of Lemma 3: Necessity is immediate. To show sufficiency, recall that $G(\cdot)$ is logconcave $\left(\ln G(\cdot)\right.$ is concave), so that $g(\cdot) / G(\cdot)>0$ and $\frac{\partial}{\partial u_{0}}(g(\cdot) / G(\cdot))<0$. The term $\pi(t, a)$ is clearly decreasing in $t$. For a fixed $a$ the last term of (3.4) is independent of $t$, so the RHS of that condition decreases in $t$ while the LHS increases. Hence $t^{*}$ is unique, given some fixed $a$. We already know that (3.5) is sufficient from the standard problem under the conditions of the FOA.

Proof of Lemma 4: Rewrite (3.4) as

$$
\frac{g(U)}{G(U)} \int_{\mathcal{Q}} u^{\prime}(t(x)) d F(x \mid a) \pi(t, a)+\mu \int_{\mathcal{Q}} u^{\prime}(t(x)) d F_{a}(x \mid a)=1
$$

where $\mu \int_{\mathcal{Q}} u^{\prime}(t(x)) d F_{a}(x \mid a)$ and $\int_{\mathcal{Q}} u^{\prime}(t(x)) d F(x \mid a) \pi(t, a)$ are all well defined and bounded. Therefore the ratio $g\left(U^{*}\right) / G\left(U^{*}\right)$ is also bounded, and $U\left(t^{*}, a^{*}\right)>\underline{u}_{0}$.

Proof of Lemma 5: Suppose $t^{*}(q)<t^{S B}(q) \forall q$ and consider a realization $u_{0}<u_{0}^{*}$. With $a$ fixed,

$$
c\left(a^{*}\right) \leq \int_{Q} u\left(t^{*}(x)\right) d F(x \mid a)<\int_{Q} u\left(t^{S B}(x)\right) d F(x \mid a)=c\left(a^{S B}\right)
$$

which contradicts the premise that $a^{*}=a^{S B}$ (i.e. $a$ is fixed). Conversely, with $t^{*}(q)>t^{S B}(q) \forall q$

$$
\int_{Q} u\left(t^{*}(x)\right) d F(x \mid a)>\int_{Q} u\left(t^{S B}(x)\right) d F(x \mid a)=c\left(a^{S B}\right)=c\left(a^{*}\right)
$$


as claimed.

Proof of Proposition 1: Uniqueness of $U^{*}$ follows directly from the fact that (3.4) and (3.5) are necessary and sufficient. The rest of the claim follows from the definition of the hazard rate $g\left(u_{0}^{*}\right) / G\left(u_{0}^{*}\right)$.

Proof of Proposition 2: Let $L(a) \equiv \int_{\mathcal{Q}}[x-t(x)] d F_{a}(x \mid a)+\mu\left[\int_{\mathcal{Q}} u(t(x)) d F_{a a}(x \mid a)-c^{\prime \prime}(a)\right]$ for some transfer function $t$. The first-order condition defining $a^{*}$ is given by $L\left(a^{*}\right)=0$ and $L^{\prime}(a) \leq 0$ (strictly here thanks to the FOA assumptions).

Suppose that the contract $\left\langle t^{*}, a^{*}\right\rangle$ is such that $a^{*} \geq a^{S B}$. Now $a^{*} \geq a^{S B}$ only if $L\left(a^{*}\right) \geq L\left(a^{S B}\right)$ at $a^{S B}$. Recalling that

$$
\int_{\mathcal{Q}} t(x) d F(x \mid a) \equiv t(a)
$$

it then follows from Lemma 5 that $t^{*}(a)>t^{S B}(a)$ for any given $a$, and therefore that $L^{*}(a)<$ $L^{S B}(a) \forall a$ (by simple substitution of $t(a)$ in $L(a)$ ). Hence the starting premise is incorrect: there cannot be a contract $\left\langle t^{*}, a^{*}\right\rangle$ is such that $a^{*} \geq a^{S B}$. Therefore $a^{*}<a^{S B}$. We know that $t(a)$ in increasing and concave (for $t(q)$ is increasing, concave in $q$ (Lemma 1 in Conlon, 2008)). It then follows that

$$
\mathbb{E}_{q}\left[t^{*}(q)\right] \equiv t\left(a^{*}\right)<\mathbb{E}_{q}\left[t^{S B}(q)\right] \equiv t\left(a^{S B}\right) .
$$

To show that $t^{*}(q)$ lies every where below $t^{S B}(q)$, I call on the monotonicity of $t(a)$; therefore there exists a function $\phi$ such that

$$
t(q) \equiv \phi(t(a))
$$

where $\phi$ is increasing. Because $a^{*}<a^{S B}, t\left(a^{*}\right)<t\left(a^{S B}\right)$ so that

$$
t^{*}(q) \equiv \phi\left(t\left(a^{*}\right)\right)<t^{S B}(q) \equiv \phi\left(t\left(a^{S B}\right)\right)
$$

Proof of Corollary 1: Let $a^{\prime}$ solve (3.1) (the agent's first-order condition in the standard problem). Now observe that because $a^{*}=\hat{a}<a^{S B}=a^{\prime}$,

$$
\int_{\mathcal{Q}} u\left(t^{*}(x)\right) d F_{a}(x \mid \hat{a})<\int_{\mathcal{Q}} u\left(t^{S B}(x)\right) d F_{a}\left(x \mid a^{S B}\right)
$$

necessarily from the agent's first-order condition. Also, $F\left(q \mid a^{S B}\right)$ FOSD $F\left(q \mid a^{*}\right)$. It is therefore also true that $F\left(q \mid a^{S B}\right)$ SOSD $F\left(q \mid a^{*}\right)$. Define the variable $q^{*}=q^{S B}+\epsilon$, where $q^{*} \sim F\left(q \mid a^{*}\right)$ and 
$q^{S B} \sim F\left(q \mid a^{S B}\right)$ (so $q^{*}$ is more risky than $\left.q^{S B}\right)$. Consider again (3.1), as under $F\left(q \mid a^{S B}\right)$, and differentiate with respect to $\epsilon$ at $\epsilon=0$ :

$$
\left[\int_{\mathcal{Q}} u(t(x)) d F_{a a}-c^{\prime \prime}(a)\right] \frac{d a^{\prime}}{d \epsilon}+\frac{d}{d \epsilon} \int_{\mathcal{Q}} u(t(x)) d F_{a}=0
$$

The last term is equivalent to (6.1) so it is negative. The bracketed one is the agent's second-order condition; it also negative. Therefore $\frac{d a^{\prime}}{d \epsilon}<0$ necessarily and so is the reciprocal. Last, take $U(a)$ and differentiate with respect to $a$ :

$$
\frac{d U\left(a, u_{0}\right)}{d a}=\left(\int_{\mathcal{Q}} u(t(x)) d F(x \mid a)-c^{\prime}(a)\right)+\frac{d \epsilon}{d a} \frac{\partial}{\partial \epsilon} \int_{\mathcal{Q}} u(t(x)) d F(x \mid a)>0
$$

where the first term is zero by (3.3) and the partial derivative necessarily negative by SOSD. So as the action decreases, so does the rent $U(a)$. Because the marginal participating agent is identified by $U\left(a, u_{0}^{*}\right)=u_{0}^{*}$ and the measure of participating agents is $G\left(U^{*}\right)$, the latter necessarily decreases as $\epsilon$ increases.

Proof of Proposition 3: First let $\hat{a}$ solve the agent's moral hazard constraint (3.3). In equilibrium, $\hat{a}=a^{*}$. Differentiate (3.3) with respect to $t$ :

$$
\int_{\mathcal{Q}} u^{\prime} d F_{a}(x \mid a)+\left[\int_{\mathcal{Q}} u(t(x)) d F_{a a}(x \mid a)-c^{\prime \prime}(a)\right] \frac{d \hat{a}}{d t}=0
$$

Since the term in the brackets is the agent's second-order condition, it is negative. Therefore $\frac{d \hat{a}}{d t}>0$.

To prove the first set of claims, consider two distributions $F^{1}(q \mid a)$ and $F^{2}(q \mid a)$, where $F^{2}$ is a mean-preserving spread of $F^{1}$ (see Rothschild and Stiglitz, 1970). Fix $t$; because $F^{1}$ dominates $F^{2}$ in the second order sense, it follows from (3.3) that at $\hat{a}$

$$
\int_{\mathcal{Q}} u(t(x)) d F_{a}^{2}(x \mid a)<\int_{\mathcal{Q}} u(t(x)) d F_{a}^{1}(x \mid a)
$$

from (6.1). Define the variable $q_{2}=q_{1}+\epsilon$, where $q_{2} \sim F^{2}$ and $q_{1} \sim F^{1}$ (so $q_{2}$ is more risky than $q_{1}$, and (6.3) follows). Consider again (3.3), as under $F^{1}$, and differentiate with respect to $\epsilon$ at $\epsilon=0$ :

$$
\left[\int_{\mathcal{Q}} u(t(x)) d F_{a a}^{1}(x \mid a)-c^{\prime \prime}(a)\right] \frac{d \hat{a}}{d \epsilon}+\frac{d}{d \epsilon} \int_{\mathcal{Q}} u(t(x)) d F_{a}^{1}=0
$$

By (6.3) the last term is negative, so $\frac{d \hat{a}}{d \epsilon}<0$. Letting $\frac{d \hat{a}}{d \epsilon} \equiv \frac{d \hat{a}}{d t} \frac{d t}{d \epsilon}$ and using $\frac{d \hat{a}}{d t}<0, \frac{d t}{d \epsilon}<0$ as claimed. To show that participation decreases, take $U(a)$ and differentiate with respect to $\epsilon$ :

$$
\frac{d U\left(a, u_{0}\right)}{d \epsilon}=\left(\int_{\mathcal{Q}} u(t(x)) d F(x \mid a)-c^{\prime}(a)\right) \frac{d a}{d \epsilon}+\frac{\partial}{\partial \epsilon} \int_{\mathcal{Q}} u(t(x)) d F(x \mid a)<0
$$


where the first term is zero by (3.3) and the second one necessarily negative by SOSD. Because the marginal participating agent is identified by $U\left(a, u_{0}^{*}\right)=u_{0}^{*}$ and the measure of participating agents is $G\left(U^{*}\right)$, the latter necessarily decreases as $\epsilon$ increases.

To show the impact of a change in risk aversion, consider a family of utility functions $u(t ; r)$ parametrized by $r$; risk aversion (i.e. the concavity of $u(\cdot ; \cdot))$ increases in $r$. Suppose for simplicity that $u(t ; r)$ is continuous and differentiable in $r$ (as well as $t$ ). For a fixed contract $\mathcal{C}$, we know that

$$
\frac{d}{d r} \int_{\mathcal{Q}} u(t(x) ; r) d F(x \mid a)<0 .
$$

That is, equivalently, for any two $r_{2}>r_{1}, \int_{\mathcal{Q}} u\left(t ; r_{2}\right) d F(x \mid a)<\int_{\mathcal{Q}} u\left(t ; r_{1}\right) d F(x \mid a)$. It then follows from (3.3) that $a^{*}\left(r_{2}\right)<a^{*}\left(r_{1}\right)$; equivalently, differentiating (3.3)

$$
\frac{d}{d r} \int_{\mathcal{Q}} u(t ; r) d F_{a}(x \mid a)+\frac{d a}{d r}\left(\int_{\mathcal{Q}} u(t ; r) d F_{a a}(x \mid a)-c^{\prime \prime}(a)\right)=0
$$

Because the first term of (6.4) is negative it follows that $\frac{d a}{d r}<0$ as well. Making use of the fact that $\frac{d a}{d t}>0$ completes the argument. To extend the result to the measure of participating agents $G\left(u_{0}\right)$ simply apply the same argument as for the SOSD claim.

Proof of Proposition 4: Because $G^{1}$ FOSD $G^{2}, G^{1} \leq G^{2} \forall u_{0} \in \mathcal{U}_{0}$ (strictly for at least a positive-measure subset of $\left.\mathcal{U}_{0}\right)$ and $\ln \left(G^{1}\right) \leq \ln \left(G^{2}\right)$ as well. Therefore by log-concavity of $G^{i}, g^{1} / G^{1}>g^{2} / G^{2}$. Immediately from the first-order condition (3.4) we must have $t^{1}$ solving it under $G^{1}$ larger than $t^{2}$ solving (3.4) under $G^{2}$ (for a fixed action). Therefore $a^{1}$ solving (3.5) under $G^{1}$ must be lower than $a^{2}$ solving (3.5) under $G^{2}$. By application of the proof of Proposition 3 (above), participation is therefore lower - i.e. because $a^{*}=\hat{a}, F\left(q \mid a^{2}\right)$ FOSD $F\left(q \mid a^{1}\right)$, which is sufficient for SOSD as well. To show the second claim, let $G^{2}$ be a mean-preserving spread of $G^{1}$ and let $u_{0}^{\prime}$ denote the value of the random variable such that $G^{2}\left(u_{0}^{\prime}\right)=G^{1}\left(u_{0}^{\prime}\right)$. Therefore

$$
\begin{aligned}
& G^{1}\left(u_{0}\right)<G^{2}\left(u_{0}\right) ; u_{0}<u_{0}^{\prime} \\
& G^{1}\left(u_{0}\right)>G^{2}\left(u_{0}\right) ; u_{0}>u_{0}^{\prime}
\end{aligned}
$$

By log-concavity of $G^{i}$, it then follows that

$$
\begin{aligned}
& g^{1} / G^{1}>g^{2} / G^{2} ; u_{0}<u_{0}^{\prime} \\
& g^{1} / G^{1}<g^{2} / G^{2} ; u_{0}>u_{0}^{\prime}
\end{aligned}
$$


and that $t^{*}$ solving (3.4) is higher (lower) when $\tilde{u}_{0}<u_{0}^{\prime}\left(\tilde{u}_{0}>u_{0}^{\prime}\right)$. Therefore $a^{*}$ solving (3.5) is lower (higher) when $\tilde{u}_{0}<u_{0}^{\prime}\left(\tilde{u}_{0}>u_{0}^{\prime}\right)$. When $a^{*}$ is lower (higher), participation is lower (higher), by application of the proof of Proposition 3 .

\section{References}

[1] Araujo A. and H. Moreira (2001) "A general Lagrangian approach for non-concave moral hazard problems. "Journal of Mathematical Economics, Vol. 35, pp. 17-39

[2] Bizer, D. and Peter DeMarzo (1992) "Sequential Banking." Journal of Political Economy, Vol. 100, pp. 41-61

[3] Conlon, John R. (2009) "Two New Conditions Supporting the First-Order Approach to Multisignal Principal-Agent Problems." Econometrica, Vol. 77(1), pp. 249-278

[4] Conlon, John R. (2008) "Supplement to Two New Conditions Supporting the First-Order Approach to Multisignal Principal-Agent Problems." mimeo, University of Mississippi.

[5] Holmström, B. (1979) "Moral hazard and observability." The Bell Journal of Economics, Vol. 10, pp. $74-91$

[6] Holmström, B. (1982) "Moral hazard in teams. " The Bell Journal of Economics, Vol. 13, pp. 324-340.

[7] Holmström, B. and Paul Milgrom (1987) "Aggregation and Linearity in the Provision of Intertemporal incentives." Econometrica, Vol. 55, pp. 303-328

[8] Holmström, B. and Paul Milgrom (1987) "Multitask Principal-Agent Analyses: Incentive Contracts, Asset Ownership, and Job Design." Journal of Law, Economics, E Organization, Vol. 7, pp. 24-52.

[9] Itoh, H. (1991) "Incentives to Help in Multi-Agent Situations," Econometrica, Vol. 59, pp. 611-636.

[10] Jewitt, I. (1988) "Justifying the first-order approach to principal-agent problems." Econometrica, Vol. 56, pp. 1177-1190 
[11] Jullien, B. (2000) "Participation Constraints in Adverse Selection Models." Journal of Economic Theory, vol. 93, pages 1-47

[12] Laffont J.-J. and David Martimort (2002) "The Theory of Incentive - the Principal-Agent model." Princeton University Press

[13] Laffont J.-J. and Jean Tirole (1986) "Using cost observation to regulate firms." Journal of Political Economy, Vol. 94, pp. 614-641

[14] Lewis, T. and David Sappington (1989) "Countervailing incentives in agency problems," Journal of Economic Theory, vol. 49(2), pages 294-313

[15] Maggi G. and Andres Rodriguez-Clare (1995) "On Countervailing Incentives," Journal of Economic Theory, vol. 66(1), pages 238-263.

[16] Martimort, D. (2004) "Delegated Common Agency under Moral Hazard and the Formation of Interest Groups." IDEI working papers

[17] Mirrlees, J. (1975,1999) "The Theory of Moral Hazard and Unobservable Behavior: Part I." The Review of Economic Studies, Vol. 66, pp 3-22.

[18] Rogerson, W. (1985b) "The first-order approach to principal-agent problems." Econometrica, Vol. 53, pp. 1357-1368.

[19] Rothschild, M. and Joseph Stiglitz (1970) "Increasing Risk I: a Definition. "Journal of Economic Theory, Vol. 2, pp. 315-329

[20] Tirole, J. "Hiearchies and Bureaucracies: On the Role of Collusion in Orgnizations. "Journal of Law, Economics and Organization, Vol. 2, 181-214 